\title{
A Catalogue of “optimization scenarios” to enhance decision-making in establishing an efficient energy management programme
}

\author{
Alfio Galata [1], Federico Di Gennaro [2], Gianfranco Pedone [3], Ya Roderick [4], Mike \\ Brogan [5] \\ [1] Agenzia per l'Energia e lo Sviluppo Sostenibile, Modena, Italy \\ [2] D’Appolonia - Gruppo RINA, Genova, Italy \\ [3] Magyar Tudomanyos Akademia Szamitastechnikai es Automatizalasi Kutato Intezet, Budapest, Hungary
}

[4] Integrated Environmental Solutions Limited, Glasgow, United Kingdom

[5] Enerit Limited, Galway, Ireland

\begin{abstract}
This paper describes how to implement an effective Energy Management Programme according to the ISO 50001 international standard, gathering the experience from the VERYSchool project. A collection of suitable "optimized scenarios" (OS), conceptualized for everyday Energy Action Management and customized for schools building and organizations has been developed, resulting in a "Catalogue" of the most common and cost effective technical and behavioural energy conservation measures. The OS Catalogue is a core component in the development of VSNavigator, a web based Energy Navigation tools. The approach focuses on client and building needs and it considers best practices to enhance energy efficiency, indoor comfort and savings. The methodology for developing and creating the OS Catalogue is transferrable to the entire European building stock (e.g. offices, residential, commercial, industrial, sport facility, hospitals, and so on): what would change are simply the OS that make any building class or typology unique (usage, energy sources, etc.).
\end{abstract}

\section{ENERGY MANAGEMENT SYSTEM (ENMS)}

Changing today's patterns of energy use in buildings is a big challenge faced with both decisionmaking and management procedures, how can organisations tap into energy efficiency potential using innovative measures designed to raise the energy performance and to help people to radical change the way they take decisions and/or interact with building energy infrastructures.

The optimal organization of the management process and the rational use of services play a key role in both increasing energy efficiency and reducing building running costs. When planning, designing and implementing an effective Energy Management System (EnMS) the ISO 50001 international standard might be the focal point to establish an effective coordination of action management constantly updated to changing needs.

The levels of services that an EnMS introduces are parameters to evaluate its effectiveness but the following principles are fundamental for an effective EnMS: indoor comfort maintained only where it is necessary and when it is strictly necessary through scaled up/down environmental conditions. An EnMS controls all the aspects involved in energy management, including technical and non-technical features that directly (or not) influence energy consumption.

\section{THE OS CATALOGUE.}

The development of technical solutions and educational and awareness campaigns are crucial in reaching energy and cost saving targets as well as to promote an energy policy rather than performing a simple energy audit.

VERYSchool proposes a software solution for establishing and maintaining an effective EnMS that covers all level of services. It is VSNavigator. It has been designed and developed to realize an ISO 50001 energy action management tool, coupled with performance monitoring system and building energy simulations. Optimization Scenarios (OS) are the mean by which the user is driven through possible implementation actions or evaluation of suitable measures.

In the VSNavigator, OS's are actions that lead to energy efficiency. Other names for OS's, are Energy Savings Strategies (ESSs), Energy Conservation Measures (ECMs), Energy Conservation Opportunities (ECOs) or Energy Efficient Retrofits (EERs).

Thus, OS's are intended as a methodology to define everything necessary for knowing how: to get starting; to get effective with the on-going process (long-medium term); to act quickly to optimize the on-going process (short-term); decisions and actions performed have been effective; (a priori) a better use 
of resources or planned investment, can introduce benefits, and so on. OS's typical examples applicable to the needs of the EnMS include:

- tariff analysis: is a contracted power rate optimal for the facility consumption patterns?

- peak violations: does a facility violate its contracted power amount?

- heating schedules: what is happening when the facility is not in use? What should be happening?

- lighting schedules: can be controlled manually, by set points, or by localized sensors? What is present? What should be present? What is the cost benefit analysis?

- what is the hot water consumption schedule? Are water temperatures or volumes adjusted given the occupancy and scheduling of the school?

- what are the heating, cooling and ventilation schedules? Are they optimal and do they actively account for the weather and building occupancy?

- what energy conservation measures are most appropriate? What renewable technology options are most appropriate?

This above list is not exhaustive but a collection of OS's, the "OS Catalogue" has to be expanded to reflect the needs of the specific building category and related organization methods (building context and territorial network). The "OS Catalogue" is a key tool to significantly increase the average range of actions that decision makers, energy managers and other key players undertake within the energy management process.

The VERYSchool project capyured the need and implemented into VSNavigator an "OS Catalogue" customized for school environments and organizations. Figure 1 shows the "OS Catalogue", with all the frameworks to support school stakeholders in planning, financing, operating, and maintaining high performing energy efficient schools.

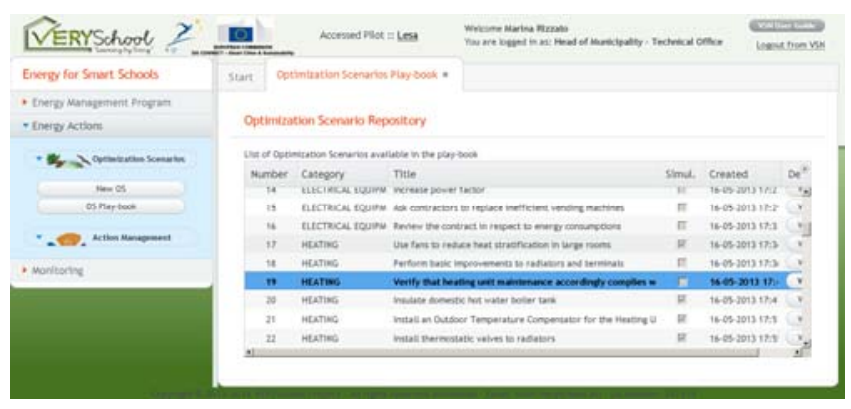

Figure 1 - the OS Catalogue

Each individual scenarios includes existing best practices, regulations (specific to schools, e.g. temperature and ventilation requirements), actions, devices suited for school buildings and educational facilities.

It is organized in several sections as follows.
- context: school uses and functional areas, building requirements, benchmarks, regulations and standards;

- implementation: standardized ISO process, decision making structure, action management, key roles and users;

- technology selection: smart metering, building management system, smart lighting, energy simulation;

- policy and regulation: rules within the organization, norms and directives applicable at local, National and European level;

- assessment: key performance indicators (KPIs) and energy performance indicators (EPIs);

A performance criteria considers the OS's classification with the aim of maximizing efficiency and user-friendliness: thus OS have been classified according to functions, trying to gather into macrocategories all possible appropriate countermeasures. For every different function/category (ventilation, air conditioning; electrical equipment; heating; lighting; system setting strategies, building elements, layout options, sport facility, renewables, management behaviour) possible actions that should "optimize" the current situation have been identified, with qualitative details about the capital cost, operation \& maintenance cost per year, energy savings, payback period and implementation timeframe. This organization guarantees a wide usability for all users according to the different type of access. Thus, for example, within VSNavigator:

- the energy manager of the school has complete access to the "OS Catalogue",

- a school manager can only manage actions done or to do within the assigned responsibilities,

- the decision maker can access to advice on selecting ICT technologies, renewable energy options, what energy audits should look like, and how they can implement energy savings programs at the macro scale,

- a manager will have visibility on how the EnMS is performing, tracking the energy consumption of the buildings including the ability to assign tasks to conduct energy savings actions until their full implementation or completion.

\section{THE INDIVIDUAL OS}

The identification of a suitable format and structure was a shared process, passing through several review made by energy managers, school managers, technician and project partners.

The design of the OS template takes account of providing information to school managers, making at the same time this information comprehensive and exhaustive for other school users, such as techni- 
cians involved in operations and maintenance activities, and without forgetting teachers and students.

Each available OS allows the implementation of an ISO 50001 standard structure, with specific requirements for establishing, implementing, maintaining and improving the EnMS, allowing the Organization to follow a systematic approach to energy management.

The OS data sheet template includes all the information needed to identify the objective where the OS is applicable, how implement the action and the potential impact of this action.

\section{Title and number}

The title provides an easily understandable identification of the OS for the users to quickly recognize the most interesting ones for the specific case.

Numbers facilitate the OS identification and the references to other OS included in the database.

\section{Main Objectives}

This section includes a general description of the OS, highlighting the main characteristics of the action and the advantages that arise from its implementation.

If the OS includes the installation of a specific technology, this technology is further detailed by explaining its usage and operation.

\section{Applicability}

This section provides information on the application field of the OS, including the main constraints that limit its implementation.

The conditions that each OS requires for its implementation are specified as well as the OS compatibility with other OS.

Thanks to these specifications, the user can easily understand if the OS can be applied to the specific case, and the degree of efficiency introduced by that action.

It is also possible to compare the OS with others within the same category, and individuate the most cost-efficiency and suitable combinations of actions for each school.

\section{Potential Impact:}

This section focuses on all the main consequences coming from the OS implementation, such as the potential reduction of energy consumption, savings on the utility bills, and reduction of $\mathrm{CO} 2$ emissions. In particular, the potential impact is contextualized within the school environment considering the specific requirements that should be respected.

The indoor comfort is a widely specified aspect and considered in most of the OS, since an adequate level of comfort including thermal, lighting and acoustic ones, is strongly necessary for a proper conduct of school activities.

This section also includes some indicative installation and operational costs.

These information are usable for giving an idea of the economic effort which a user should face for implementing such OS. Furthermore, it is also possible to forecast the payback period of this investment, and eventually plan the retrofitting activities over time.

\section{Implementations Steps}

This section describes the main steps for implementing the OS. If the OS consists in the installation of a specific technology, there is an explanation of the installation process of this technology even structured in all its components.

To make the OS implementation maximally costefficient, and optimized over the time, best practices for planning preliminary actions are also included.

\section{Reference}

Here, the main European and National regulations are indicated, together with scientific papers and on-line publications related to that OS.

From these references, aspects regarding the OS descriptions, applicability and potential impact considerations and the list of implementation steps have been extrapolated underling all the information that can be linked to a school context.

In this way, a school user who is consulting the OS database can easily access to other sources and eventually deepen certain topics.

\section{Reference Suppliers}

This section includes a list of some suppliers of services or technologies related to the OS that have been selected from the market.

This list provides suggestions to the school users as starting point for investigating.

By contacting a supplier, it will be possible to obtain more specific details on the OS applicability, potential impacts and implementation steps, but, above all, information regarding the potential energy savings and economic effort distributed over time.

\section{Related OS}

This section combines the selected OS with the others in the Catalogue, that eventually represent a valid alternative or that are just linked to.

Aim of this information is to make the database consultation easier and faster.

In relation to specific retrofitting actions which a school need, a user can easily find a set of OS to combine or compare with each other, and individuate the most cost-efficiency combination of strategies. 
A significant picture related to the OS is depicted in Figure 2 below.

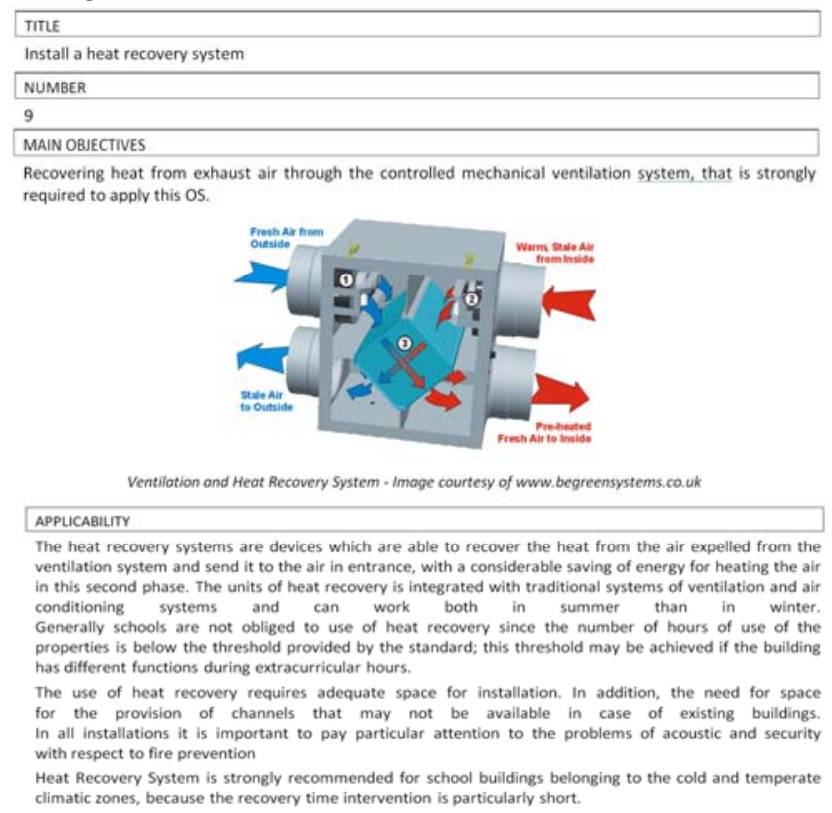

Figure 2 - the OS template

\section{DECISION SUPPORT SYSTEM: LINK OS TO SET RULES}

The OS Catalogue is not just a simple checklist of energy saving measures but an online tool of recommendations and actions that are intelligently proposed using "Set-Rules". Set Rules are the beating heart of the decision-making process within VSNavigator database. Figure 3 shows the basic concept behind set rules construction and their functioning.

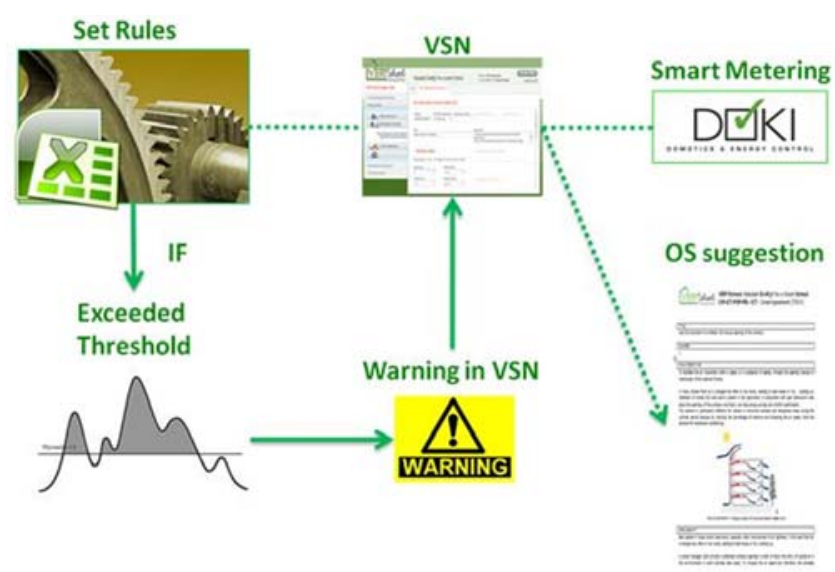

Figure 3: Set Rules diagram flow

Set rules are the mechanisms that propose specific OS in an intelligent and dynamic way. These mechanisms are made by a set of algorithms based on several parameters, called configuration data (e.g. occupancy profile, temperature, window status etc.) and related threshold (e.g. comfort level, illuminance value etc.).

Set rules use configuration data to automatically generate warning messages that further propose OS to the user (e.g. energy manager) in order to decide on the opportunity for implementing an Energy Action.

The triggers for set rules come from experimental data received by the BEMS, such as temperature or illuminance values, or data coming from energy bills and Pilot layout.

Ideally each Optimisation Scenario is associated with a specific set rule.

Heating system turned on too early in the morning ( $T=$ Tset more than 1 hour before the school opening time)

Required Data: Internal real temperatures values per hour per room

Readings: Room temperature sensors

Location: Room in the Pilot School

Computation: Actions taken in order to measure if the required temperature for school opening time is reached too early in the morning.

Check Tmax $\left[{ }^{\circ} \mathrm{C}\right] 1$ hour before school opening time and verify if Tmax $\geq$ Tset $\pm 2\left[{ }^{\circ} \mathrm{C}\right]$

Figure 4: example of Set Rule.

This set rule takes into account a quite common situation in (school) buildings: according to several interviews and meetings with energy managers, heating system is often turned on too early in the morning: this action lead to many waste in terms of energy and, consequently, money.

School managers and/or technician are not always informed of the heating system starting time: this set rule solves this issue by considering several parameters with a fixed threshold to alert if there is a malfunctioning.

To make this OS operational, the required data are the internal real temperatures values per hour per room.

Then a computation is done, considering the temperature in school rooms one hour before school opening time: if this temperature is too high or too low compared to the pre-determined threshold, a warning will be launched in VSNavigator.

\section{THE OS CATALOGUE LINKED WITH THE ISO 50001}

The OS Catalogue is the kernel of the decision support system and it helps to overcome features that may cause confusion for non-expert users on how to create an effective EnMS: these include, for example, knowledge on what technologies to select; what energy conservation measures to pursue first; how to implement these choices in a systematic way.

As research and technologies progresses with increasing velocity, people that must take deci- 
sions and act upon to meet energy reduction targets, are being left behind: options are overwhelming, timing customisation and business model are misunderstood, guidelines are not yet full standardised.

Along the value chain of stakeholders:

- Public Administrators overview cost savings, reward efforts to best energy classes, broadcast best practices and energy management rated lower in energy class.

- Operational, Energy and Facility Managers, take energy decisions to get started the energy management process or to be more effective on the current operational energy scenario.

- Technicians, have the day-by-day responsibility to maintain and operate the buildings.

- ESCO and Financial Institutions, are interested in promoting concepts of green economy where energy saving pays for investments.

- ICT and Scientific Professionals present best practices and new technologies, while producing awareness on efficient scenarios and habits.

- Practitioners want to learn about best practices on energy efficiency.

Because this value chain is not linked, energy savings actions and programs are generally not effective or not taking place.

Thus, to give value to the "OS Catalogue" it has to operate within the framework of an energy management system based on the ISO 50001standard.

Management of any suggested OS, selected by the user for implementation, is provided through the integration of the VSNavigator and the "Enerit ISO 50001 software”.

\section{DESIGN, IMPLEMENTATION AND SIMULATION PERFORMANCES}

The OS Catalogue is part of the over-all solution for school energy optimization strategies provided by VSNavigator. The design of the OS Catalogue relies on the generic data representation schema depicted in Figure 5.

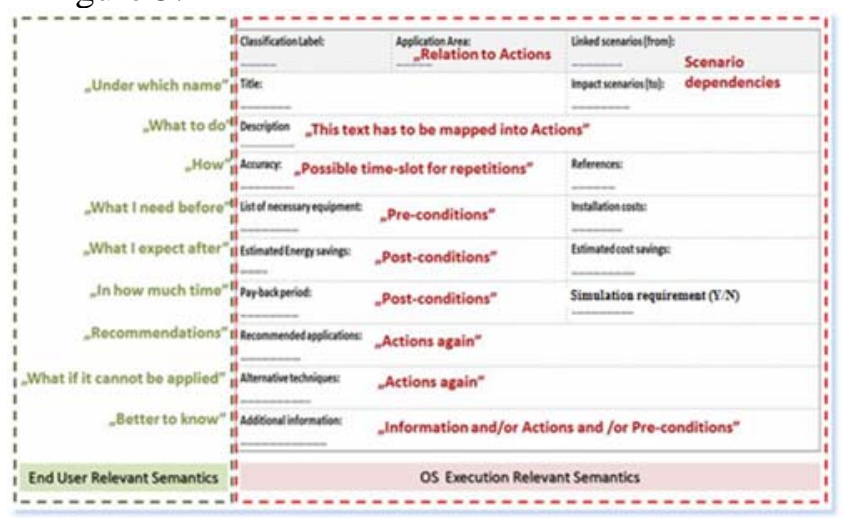

Figure 5 - the OS Catalogue representation schema
Starting from this schema, the OS persistence design is realized by a high-level Entity-Relation Diagram (ERD). To be able to assist users making the appropriate decision, each OS is analysed in terms of dynamic simulation modelling (DSM) performances.

Two main categories of OSs were specialized: the Operational OSs (relying on experimental data) and the Design OSs (based on the school generic configuration). ). Other OSs provides generic indication, although customized to all the European Countries, if no simulation is required.

From the point of view of the implementation details, the OS Catalogue is part of a much more complex web-application, and it is implemented leveraging both server and client side technology.

The core piece of the OS Catalogue (and the VSNavigator as a whole) is the Vaadin Framework, a Java library that is designed to make easy creation and maintenance of high quality web-based user interfaces.

The idea in server driven programming model of Vaadin is innovative and competitive because the developer can manage the user interface in the browser and AJAX communications between the browser and the server. Hidden under the hood, the OS Catalogue uses GWT, the Google Web Toolkit, for rendering the user interface in the browser. GWT programs are written in Java, but compiled into JavaScript.

The OS Catalogue is stored into a platform utilizing PostgreSQL 8.4 as Relation Database Management System. Being the over-all application a J2EE type and as such needing an application server to run (more specifically a servlet container), it was deploy it under the well-know Apache Tomcat 6.0, an open source software implementation of the Java Servlet and JavaServer Pages technologies.

To estimate the energy impact and the potential return on investment for those scenarios requiring simulation (operational and design categories), VSNavigator links the "IES $<\mathrm{VE}>$ performance analysis software suite”.

To have accurate building simulation results a Calibrated Model is required, specifically configured to best match actual building performances. Figure 6 shows an overview of this framework.

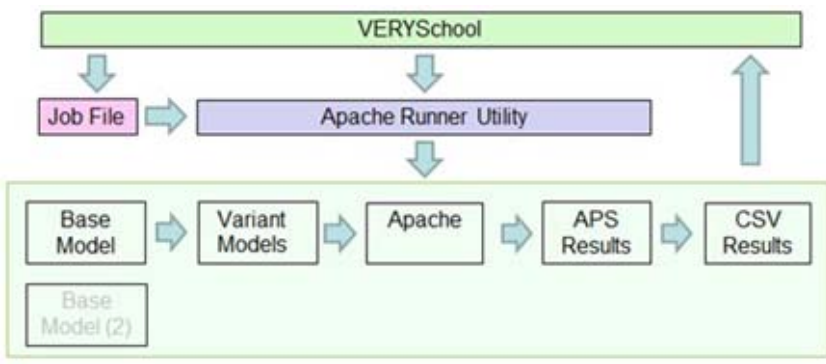

Figure 6: Overview of VSNavigator to IES $<$ VE $>$ link 
For these OS, a BATCH and JAVA programming based link has been realized among VSNavigator and the database layer and IES $<\mathrm{VE}>$ simulation engine, to daily update schools' potential energy saving strategies according to indications contemplated in operational scenarios.

Figure 7 shows an example where different setpoint configurations are evaluated against the current one with related impacts on energy consumptions, carbon emission and indoor comfort conditions.

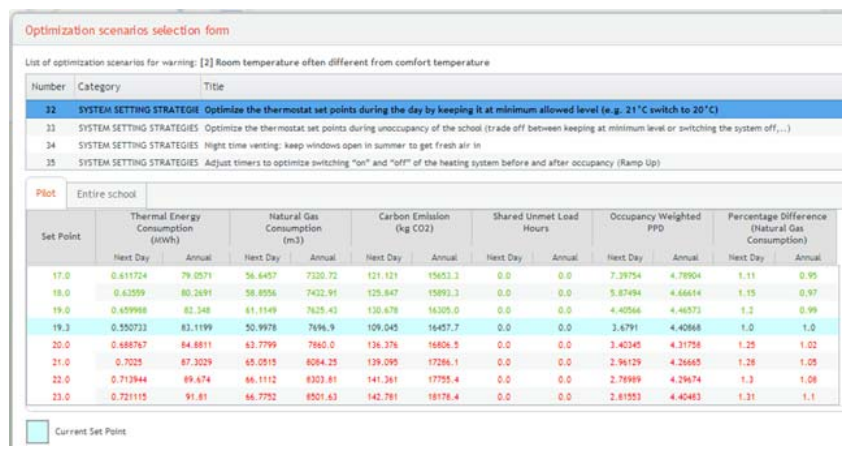

Figure 7: Simulation results for a particular OS.

\section{RESULTS}

The online OS Catalogue along with associated setrules have already been successfully developed for schools within the VERYSchool project and it is under testing (demonstration) and validation.

Preliminary results achieved through the IPMVP methodology on thermal consumptions shows energy savings ranging between $24 \%$ to $40 \%$, and on average the indoor thermal comfort is well maintained. Savings are achieved through a strategy on set-point values implemented through the EnMS.

The methodology here described introduce innovation in energy management by proposing an "Energy Action Navigator", called VSNavigator, to Organizations which simply need a baseline to get where they are going or where they want to go. VSNavigator, as a product and as a service, can be easily extend to all the building stocks and energy infrastructure, rather than for only school buildings and organization.

\section{CONCLUSIONS}

A systematic approach for systematically establishing an energy management program based on selected "optimization scenarios" from a Catalogue is a standard way for implementing most common and cost effective energy conservation measures (usage, building types and energy infrastructure), while conforming with standards.

The performance of such OS Catalogue, integrated into a decision support system, allow:
- To document all the identified Optimisation Scenarios for school's needs in terms of energy saving solutions and best practices that can bring to energy and cost savings.

- $\quad$ To use metered data for applying set-rules and to simulations, through a calibrated building model, so that indications provided for an operational or design OS are accurate.

Furthermore, the methodology describes how the OS Catalogue is linked to the VSNavigator functionality so that the OS can be directly activated by the Navigator to estimate the energy impact and the potential return on investment for those selected scenarios requiring simulation.

For "eLearning” users, a demonstration version of VSNavigator is available at the project website: http://www.veryschool.eu/news.php?id=45

\section{ACKNOWLEDGEMENT.}

The VERYSchool project (GA n 297313 for CIPPilot actions) receive funds by the EC under the ICT-PSP-CIP framework Program.

The Consortium is made by 12 Partners, which collectively contribute to achieve the project results.

\section{REFERENCES.}

Directorate General for Education and Culture - European Commission. Year 2009 - EUROSTAT Databases and eTwinning 2.0 Building the community for schools in Europe.

VERYSchool project, 2013. Deliverable D3.1 School specific optimization scenarios and its Annex $A$. In project website: www.veryschoo.eu.

The UrbanISM (Integrated Spatial Model). Testing the future: evidence-based scenario assessment. In www.urbaninitiatives.co.uk.

U.S. Department of Energy, September 2011. Advanced Energy Retrofit Guide - Practical Ways to Improve Energy Performance.

C. Onyeagor and others, August 2011. Building Retrofit Industry and Market (BRIM) Market Research Scan. The Rockefeller Foundation.

European Construction Technology Platform web site. Energy Efficient Building European Initiative.

Robinson and others, 2008: Towards comprehensive simulation and optimization for more sustainable urban design. In PLEA - 25th Conference on Passive and Low Energy Architecture, Dublin, October 2008 